\title{
Correlation with Large Transverse Momentum Photons and the Gluon Structure Function
}

\author{
R. Baier, J. Engels, and B. Petersson \\ Department of Theoretical Physics, University of Bielefeld, D-4800 Bielefeld, Federal Republic of Germany
}

Received 19 May 1980

\begin{abstract}
The inclusive two-particle cross section for the production of large $p_{T}$ photons and opposite side charged hadrons in proton-proton collisions is examined in detail in the framework of QCD. The model, with the parametrization as in our previous work, agrees with the measured single photon spectra. Quantitative predictions are made for twoparticle distributions to be measured at the ISR. The contributions due to the photon bremsstrahlung and the effects coming from the intrinsic constituent motion are estimated. It is shown that indeed the direct subprocess gluon + quark $\rightarrow$ photon + quark gives the dominant contribution in the experimentally relevant region of phase space, and that direct information about the gluon structure function can be obtained from such measurements.
\end{abstract}

\section{Introduction}

The production of real photons at large transverse momentum in hadron-hadron collisions is of particular interest for hard scattering models, because it can possibly be used as a direct probe of quark and gluon subprocesses involving the pointlike coupling of the photon to the quark. The existence, in QCD, of a gluon-photon Compton process, $g+q \rightarrow \gamma+q$, leads to the prediction that the $\gamma / \pi^{0}$ ratio is large compared to $\alpha=1 / 137$, since the photon is produced directly, whereas the $\pi^{0}$ is a fragment of a quark or gluon jet coming from the subprocesses $q+q \rightarrow q+q$ etc. For single particle spectra quantitative predictions have been made by several authors [1-9].

Recent experiments at the CERN-ISR [10-13] support at least qualitatively the hard scattering picture. The experimental single $\gamma / \pi^{0}$ ratio is indeed larger than $\alpha$ for large $p_{T}$. The measurement of the same side correlations shows that the $\pi^{0}$ is accompanied by other particles to a significantly higher degree than the photon [14].
In this paper we make, in the framework of QCD, a first quantitative analysis of the two-particle cross section $p+p \rightarrow \gamma+$ opposite side charged hadrons + anything. An experiment measuring this quantity is under way [15]. Our motivations are twofold. Firstly, in the hard scattering model the two-particle cross section may be described by a much simpler picture than the single particle yield. This is due to the suppression of Fermi motion effects [16]. Secondly, the possible dominance of the direct term, $g+q \rightarrow \gamma+q$, gives a number of qualitative expectations for the kinematic and quantum number structure of the opposite side jet.

Section II contains a short discussion of the scale breaking parametrizations of structure and fragmentation functions used for the following calculations with special emphasis on the gluon distribution function. In Sect. III the results for the single particle spectra for photons and $\pi^{0}$ 's are summarized and their comparison with data is discussed. In Sect. IV predictions for the two-particle cross section are given for $p+p \rightarrow \gamma+$ charged opposite side hadrons + anything. In addition to the dominant subprocess we also take into account the annihilation process $q+\bar{q} \rightarrow \gamma+g$ and the bremsstrahlung reactions $q+q \rightarrow \gamma+q+q$ and $q+g \rightarrow \gamma+q+g$. The possible determination of the gluon structure function from measurements of the $s$ and $p_{T}$ dependence of the two-particle cross section is examined.

\section{The Structure and Fragmentation Functions}

The parametrizations for the structure and fragmentation functions utilized in the following calculations are described in detail in our previous paper on symmetric hadron pairs at large $p_{T}$ [16]. The results were compared with FNAL data and lead to a quite successful prediction for the production of $\pi^{0}$ pairs in the ISR energy range [17].

The parametrizations of the valence and sea quark distributions were taken from the CERN- 
Dortmund-Heidelberg-Saclay Collaboration [18]. The gluon is described by the ansatz

$x G\left(x, Q^{2}\right)=c_{g}(\bar{s}) x^{\eta_{1}^{g}(\bar{s})}(1-x)^{\eta_{2}^{g_{2}(\bar{s})}}$,

$\bar{s}=\ln \left(\frac{\ln Q^{2} / \Lambda^{2}}{\ln Q_{0}^{2} / \bar{\Lambda}^{2}}\right)$.

At $Q_{0}^{2}=5 \mathrm{GeV}^{2}$ the input parameters have been determined from the second and third gluon moments as given in [18]. From the QCD moment equations [19] for these two moments we have obtained, by a quadratic fit, the following $\bar{s}$-dependence for $0 \leqq \bar{s}<1$,

$c_{g}(\bar{s})=2.01-2.73 \bar{s}+1.29 \bar{s}^{2}$,

$\eta_{1}^{g}(\bar{s})=-0.93 \bar{s}+0.36 \bar{s}^{2}$,

where as input $\eta_{2}^{g}(\vec{s})=2.9+5.08 \frac{9}{25} \vec{s}$ has been used. As a check we have evaluated the higher moments and the QCD evolution equation [19]

$x G(x, \bar{s})$

$=x G(x, 0)+\frac{36}{25} x \int_{0}^{\bar{s}} d \bar{s}^{\prime}\left\{\left[\frac{25}{36}+\ln (1-x)\right] G\left(x, \bar{s}^{\prime}\right)\right.$

$+\int_{x}^{1} d y\left[\frac{G\left(x / y, \bar{s}^{\prime}\right)-G\left(x, \bar{s}^{\prime}\right)}{1-y}\right.$

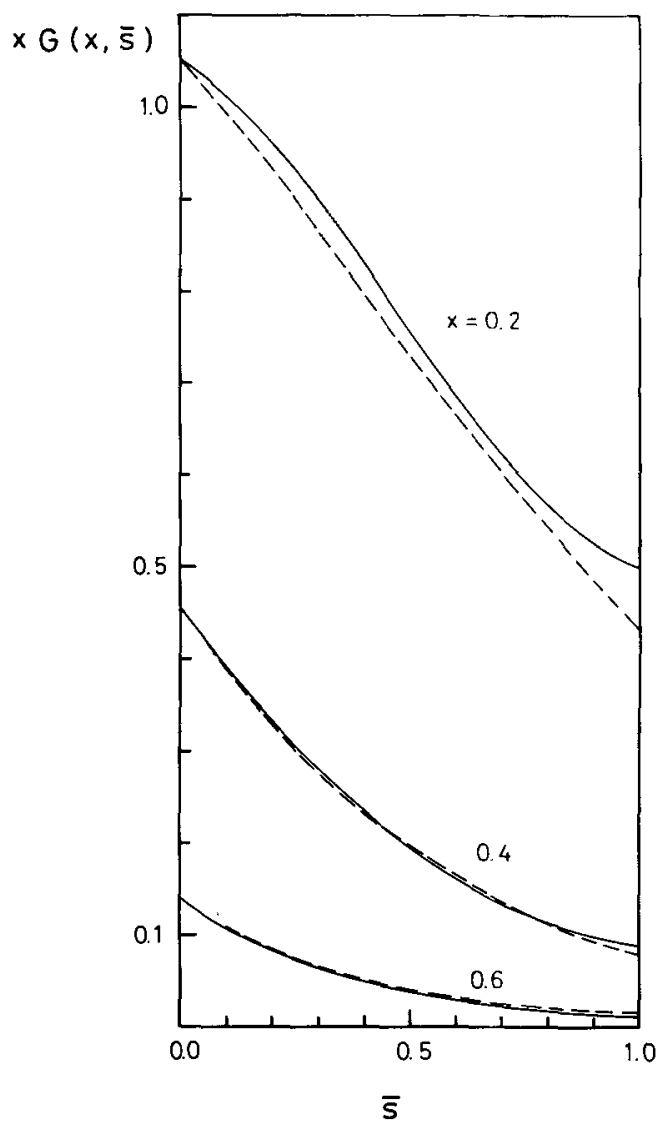

Fig. 1. The dependence on $x$ and $\bar{s}$ of the gluon structure function. The dotted curves show $x G(x, \bar{s})$ according to the parametrization described in the text. The solid curves result from $(2.3)$

$$
\begin{aligned}
& \left.+(1-y)\left(1+\frac{1}{y^{2}}\right) G\left(x / y, \bar{s}^{\prime}\right)\right] \\
& \left.+\frac{2}{9} \int_{x}^{1} d y \frac{1+(1-y)^{2}}{y^{2}}\left[q^{N S}\left(x / y, \vec{s}^{\prime}\right)+6 \bar{q}\left(x / y, \bar{s}^{\prime}\right)\right]\right\},
\end{aligned}
$$

where $q^{N S}$ and $\bar{q}$ are the non-singlet and the sea-quark distributions, respectively.

Figure 1 shows a comparison of the two sides of the above equation for $x G(x, \bar{s})$ as a function of $\bar{s}$ for different values of $x$. The full curve corresponds to the gluon distribution described above (2.1), (2.2) which is used as input into the right hand side. The corresponding output $x G(x, \bar{s})$ is the dashed curve. The two curves almost coincide. For the other distributions the test works as well.

Compared to the parametrization in [16] we have changed the coefficient of $\bar{s}$ in $\eta_{2}^{g}$. It is now $9 / 4$ times the corresponding coefficient in the valence distribution. The behaviour for $x \geq 0.6$ is thereby improved. This change does not influence our earlier results, which are not sensitive to that region in $x$. However, the input values at $\bar{s}=0$ remain unchanged implying a gluon distribution proportional to $(1-x)^{3}$ at $Q_{0}^{2}=5 \mathrm{GeV}^{2}$. This hard gluon distribution is essential for the good agreement with the data on symmetric hadron pairs $[16,17]$.

\section{The Single Particle Spectra}

There exist two experiments at the CERN-ISR, which have measured single photon as well as single $\pi^{0}$ spectra at large $p_{T}[10-12,20,21]$. The results are usually given in the form of ratios of the $\gamma$ to the $\pi^{0}$ spectra. The $\gamma$ spectrum itself is shown in [12]. Unfortunately the two experimental groups (AABC and CCOR) get different results for $\sqrt{s}=63 \mathrm{GeV}$ and $p_{T}>6 \mathrm{GeV} / \mathrm{c}$ both for the $\pi^{0}$ spectrum and the $\gamma / \pi^{0}$ ratio.

We have calculated the single $\pi^{0}$ spectra including intrinsic transverse momenta. The spectra are in good agreement with both experiments, whenever the data are compatible with each other; for $\sqrt{s}=63 \mathrm{GeV}$ and $p_{T}>6 \mathrm{GeV} / c$ they follow the results of the $\mathrm{AABC}$ group.

It is important to have a hard gluon structure function in order to describe these data. This was noted already by Field [22] and others [23].

We have computed the $\gamma$ spectrum taking into account the subprocesses $g+q \rightarrow \gamma+q$ and $q+\bar{q} \rightarrow \gamma+g$. The indirect bremsstrahlung processes have not been considered. Aurenche and Lindfors [24] showed that they contribute about $10 \%$ (using our parametrization for the structure functions). The $\gamma / \pi^{0}$ ratios are then deduced from the $\gamma$ and $\pi^{0}$ calculations within the model.

The theoretical single particle spectra turn out to be strongly dependent on the intrinsic transverse 
Table 1. $R_{\pi^{0}}$ and $R$ are the ratios of the results for $E d^{3} \sigma / d^{3} p$ with and without intrinsic transverse momenta

\begin{tabular}{llcl}
\hline$\sqrt{s}[\mathrm{GeV}]$ & $p_{T}[\mathrm{GeV} / c]$ & $R_{\pi^{0}}$ & $R_{\gamma}$ \\
\hline 19.4 & 3 & 17 & 4.2 \\
& 6 & 5.6 & 3.7 \\
\hline 31 & 3 & 6.6 & 2.3 \\
& 6 & 1.9 & 1.6 \\
\hline 44 & 3 & 4.3 & 1.8 \\
& 6 & 1.7 & 1.3 \\
\hline 63 & 3 & 3.6 & 1.5 \\
& 6 & 1.4 & 1.3 \\
\hline
\end{tabular}

momentum of partons in most of the accessible range of $p_{T}$ and $\sqrt{s}$. Only for $\sqrt{s}=63 \mathrm{GeV}$ and $p_{T} \gtrsim 6 \mathrm{GeV} / c$ the effects are less than $50 \%$. They are smaller for the $\gamma$ spectrum than for the $\pi^{0}$ spectrum, because the former is flatter. The results are summarized in Table 1 . When the effects of intrinsic trans-
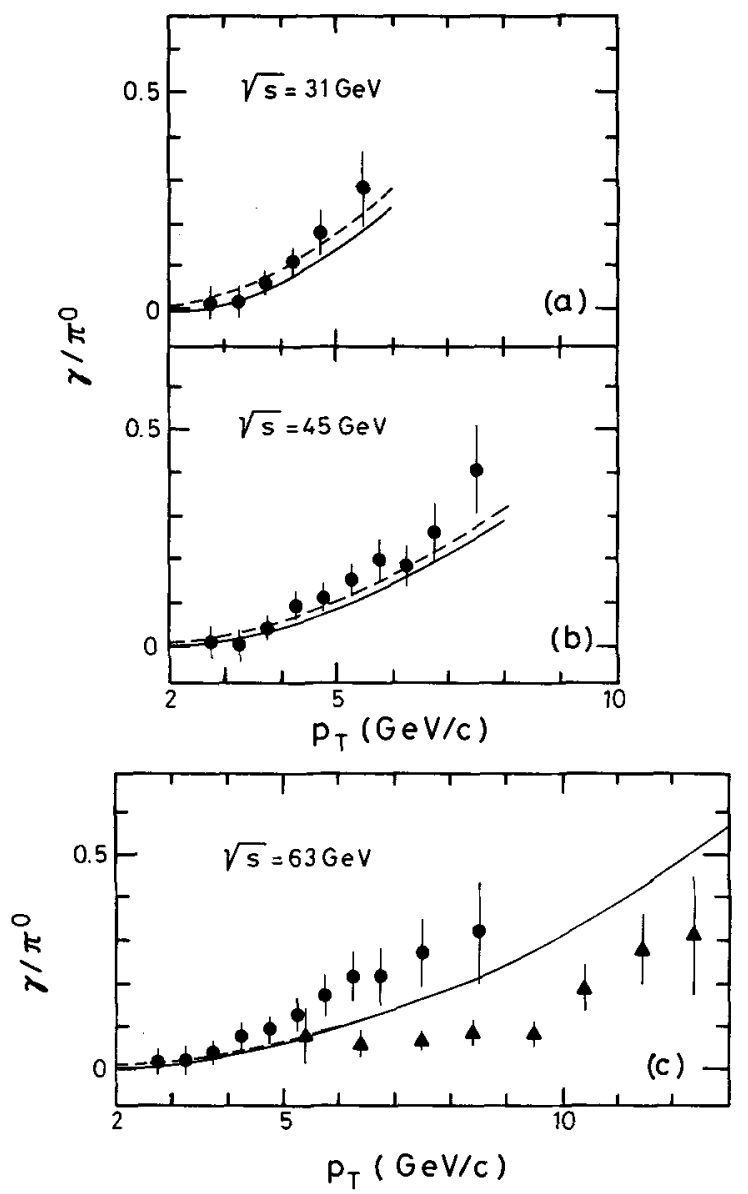

Fig. $2 \mathbf{a}-\mathbf{c}$. The $\gamma / \pi^{0}$ ratio as a function of $p_{T}$ at $\mathbf{a} \sqrt{s}=31 \mathrm{GeV}$, b $\sqrt{s}=45 \mathrm{GeV}, \mathbf{c} \sqrt{s}=63 \mathrm{GeV}$. The data points of [11] (dots) are multiplied by 0.85 , the ones of [12] (triangles) by 0.80 . The solid (dashed) curves are the calculated $\gamma / \pi^{0}$ ratios with (without) intrinsic transverse momenta verse momenta are large, one may expect that other higher twist terms are also important.

The $\gamma / \pi^{\circ}$ ratios at $90^{\circ}$ at three energies are plotted in Fig. 2. At $\sqrt{s}=31$ and $45 \mathrm{GeV}$ there is agreement with the data [11], at $\sqrt{s}=63 \mathrm{GeV}$ our ratio is smaller than the AABC data [11], but larger than the CCOR data [12]. Taking a softer gluon distribution at the input value $Q_{0}^{2}=5 \mathrm{GeV}^{2}$, e.g. proportional to $(1-x)^{5}$, would lower our curves significantly. We have checked the dependence on $\Lambda$ : the ratios are insensitive to a variation in the range $0.3 \leqq \Lambda \leqq 0.6 \mathrm{GeV} / c$.

The theoretical ratios are not a function of $x_{T}=2 p_{T} / \sqrt{s}$, because of the stronger scale breaking effects in the $\pi^{0}$ spectra, which are due to the extra power of $\alpha_{s}\left(Q^{2}\right)$ and the fragmentation function. In fact the ratios are, like the data, more close to a function of $p_{T}$ only.

In Fig. 3 we compare our calculation for $p+p \rightarrow$ $\gamma+X$ at $\sqrt{s}=63 \mathrm{GeV}$ to the data [12]. Again our curves are between the results of the two experimental groups.

In Fig. 4 we show the prediction for the Feynman $x_{F}$ dependence of the single $p+p \rightarrow \gamma+X$ cross section at $\sqrt{s}=19.4 \mathrm{GeV}$ for different values of $p_{T}$. The plot illustrates that the cross section depends strongly on the intrinsic transverse momenta. (At $p_{T}=2 \mathrm{GeV} / c$ the effects are smaller, because we have cut out contributions with $Q^{2}<4 \mathrm{GeV}^{2}$ ).

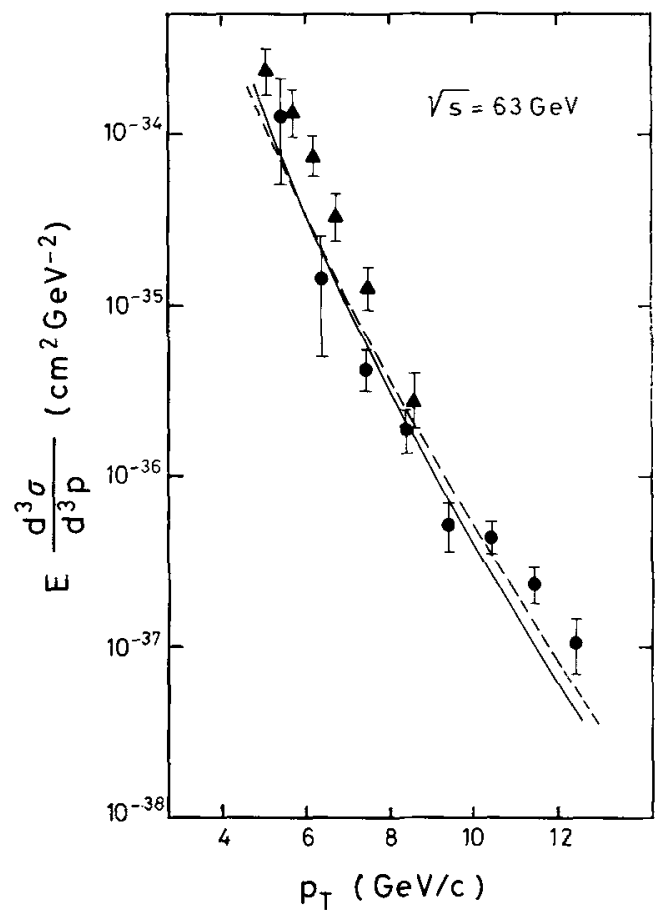

Fig. 3. The cross section for single photon production in $p-p$ collisions as a function of $p_{T}$ at $\sqrt{s}=63 \mathrm{GeV}$. The data points are taken from [12]. The solid (dashed) curve shows the result of our QCD calculation with $A=0.6(0.3) \mathrm{GeV}$ 


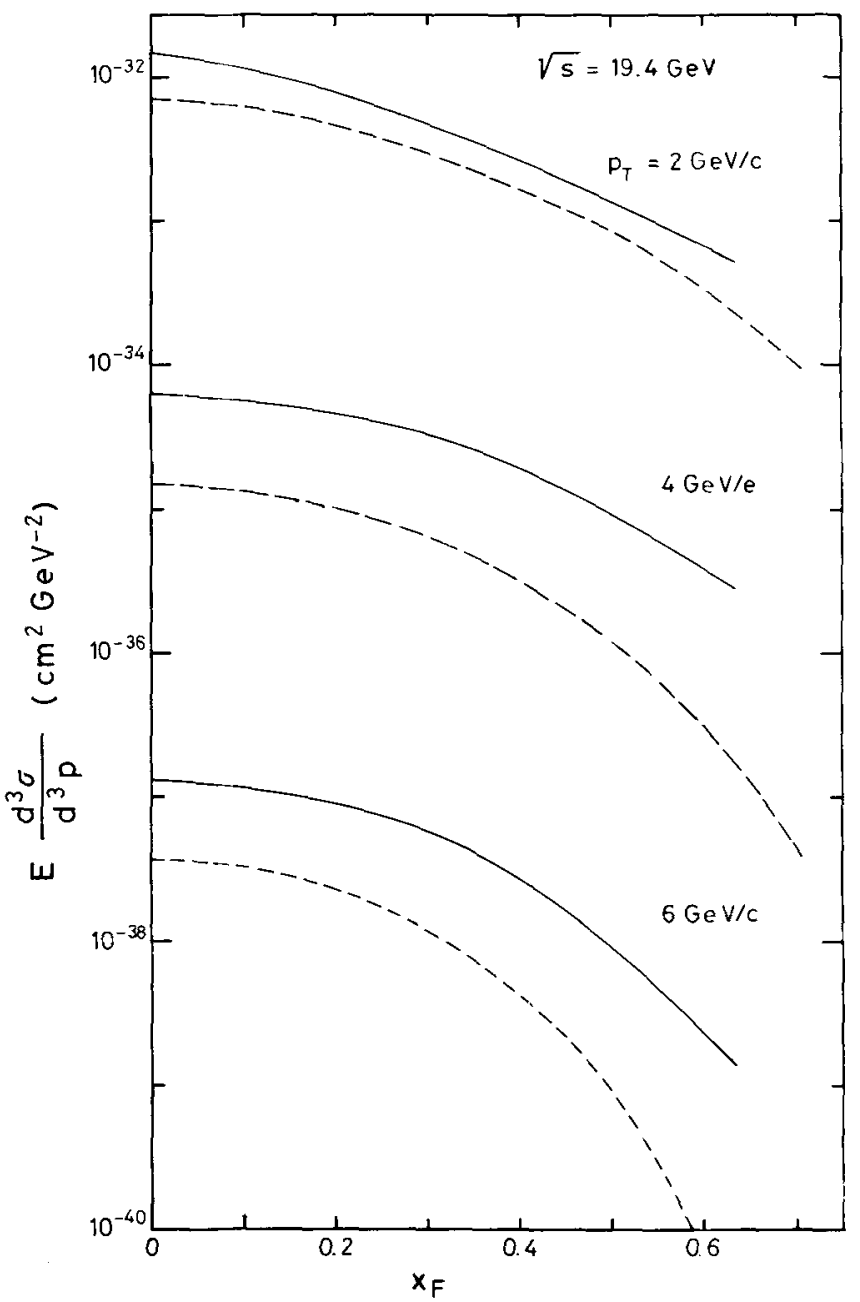

Fig. 4. The $x_{F}$-dependence of the cross section $E d^{3} \sigma / d^{3} p$ for $p+p \rightarrow y+X$ at $\sqrt{s}=19.4 \mathrm{GeV}$ for $p_{T}=2,4$ and $6 \mathrm{GeV} / c$. The solid (dashed) curves are calculated with (without) intrinsic transverse momenta

Furthermore, in the inclusive spectra the gluon distribution is integrated over the range $x_{\min }\left(x_{F}, x_{T}\right) \leqq$ $x \leqq 1$. Therefore we believe that it is not appropriate to deduce the gluon distribution from them.

\section{The Two-Particle Spectra}

The study of the reaction $p+p \rightarrow \gamma+h+X$ offers the exiting possibility of a direct measurement of the gluon structure function. When the photon and the hadron are produced at $90^{\circ}$ in opposite hemispheres, the cross section in the hard scattering limit from the subprocess $g+q \rightarrow \gamma+q$ is given by

$$
\begin{aligned}
& \left.E_{\gamma} \frac{d^{5} \sigma}{d^{3} p_{\gamma} d x_{E} d y_{h}}\right|_{y_{\gamma}=y_{h}=0}=\frac{2}{\pi} x G\left(x, Q^{2}\right) F\left(x, z, Q^{2}\right) \\
& \cdot\left(\frac{1}{e_{q}^{2}} \frac{d \sigma}{d \hat{t}}(g+q \rightarrow \gamma+q)_{90^{\circ}}\right),
\end{aligned}
$$

where

$\frac{1}{e_{q}^{2}} \frac{d \sigma}{d \hat{t}}=\frac{\pi \alpha \alpha_{s}\left(Q^{2}\right)}{3 \hat{s}^{2}}\left(-\frac{\hat{s}}{\hat{t}}-\frac{\hat{t}}{\hat{s}}\right)$,

and $e_{4}$ is the quark charge, $\alpha$ the electromagnetic and $\alpha_{s}\left(Q^{2}\right)$ the strong coupling constant. At $90^{\circ}$ one has $x=x_{T}=2 p_{T \gamma} / \sqrt{s}, \hat{s}=x_{T}^{2} s, \hat{t}=\hat{u}=-\hat{s} / 2$ and $z=$ $x_{E}=p_{T h} / p_{T_{y}}$. In the leading logarithm approximation [25] the function $F$ is related to the quark structure function $q\left(x, Q^{2}\right)$ and to the fragmentation function $D_{q}^{h}\left(z, Q^{2}\right)$ through

$F\left(x, z, Q^{2}\right)=\sum_{q, q} e_{q}^{2} x q\left(x, Q^{2}\right) D_{q}^{h}\left(z, Q^{2}\right)$.

Because of the factorization property in this approximation [25], the function $\mathrm{F}$ can be taken directly from the deep inelastic cross section for the process $\mu+p \rightarrow \mu+h+X$. The quantity $Q^{2}$ is a combination of the invariants $\hat{s}, \hat{t}$ and $\hat{u}$; we have taken $Q^{2}=2 \hat{s} \hat{t} \hat{u} /\left(\hat{s}^{2}+\hat{t}^{2}+\hat{u}^{2}\right)$ as in [26].

Modifications to the simple (4.1) may come from the following sources:

(i) fusion processes $q+\bar{q} \rightarrow \gamma+g$;

(ii) inclusion of intrinsic transverse momenta;

(iii) indirect photons from bremsstrahlung corrections to elastic quark-quark and quark-gluon scattering;

(iv) rapidity dependence of the cross section, when comparison is made with data which are not exactly at $90^{\circ}$.

The fusion processes contribute less than $10 \%$ for the results to be presented, because of the small size of the sea quark distributions. The effects due to the intrinsic transverse momenta and the bremsstrahlung terms might be important at large $x_{E}$, since the direct process vanishes according to (4.1) and (4.3) at $x_{E}=1$, if $D_{q}^{h}\left(z, Q^{2}\right)$ vanishes for $z \rightarrow 1$.

We have followed the conventional on-mass shell approach in order to include intrinsic transverse momenta $[22,26]$. The resulting formula has been evaluated by the Monte Carlo method. It turned out that to a very good approximation the following analytic expression can be used

$$
\begin{aligned}
& \left.E_{\gamma} \frac{d^{5} \sigma}{d^{3} p_{\gamma} d x_{E} d y_{h}}\right|_{y_{\gamma}=y_{h}=0} \\
& =\frac{2 p_{T \gamma}}{\pi} \int_{x_{T}^{2} x_{E}}^{1} \frac{d z}{\exp \left[-\frac{p_{T \gamma}^{2}\left(1-x_{E} / z\right)^{2}}{2\left\langle q_{T}^{2}\right\rangle}\right]} \\
& \cdot x G\left(x=\sqrt{2 \pi\left\langle q_{T}^{2}\right\rangle}\right. \\
& x \in\left(\frac{1}{s}, Q^{2}\right) F\left(x, z, Q^{2}\right)\left(\frac{1}{e_{q}^{2}} \frac{d \sigma}{d \hat{t}}\right)_{90^{\circ}},
\end{aligned}
$$

where $\hat{s}=4 p_{T \gamma}^{2} / z$. We have taken the same values of the parameters as in [16], 


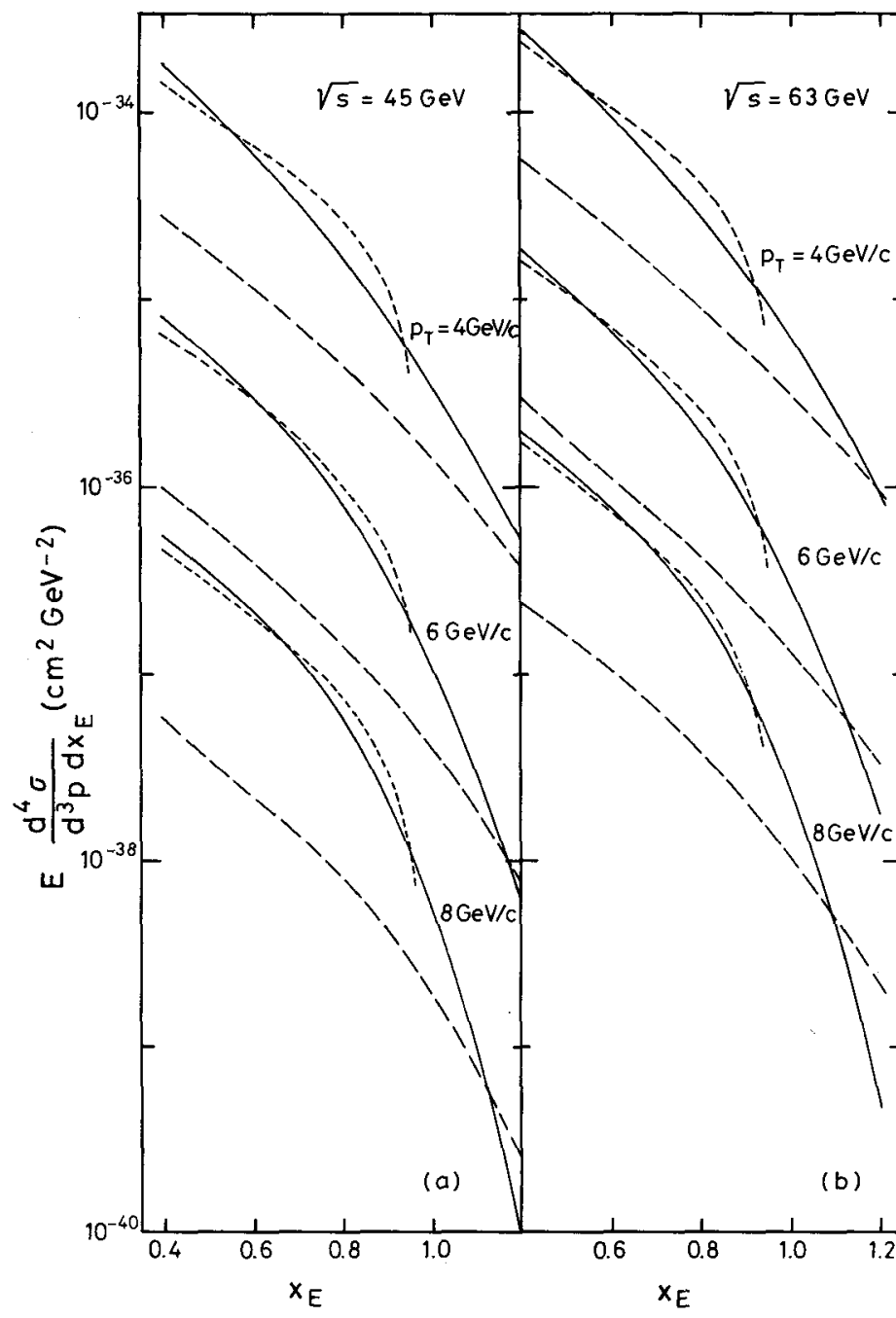

Fig. 5a and b. The $x_{E}$-dependence of the cross section $E d^{4} \sigma / d^{3} p d x_{E}$ for $p+p \rightarrow \gamma+h^{+} X$ for $p_{T}=4,6$ and 8 $\mathrm{GeV} / c$ at $\sqrt{s}=45 \mathrm{GeV}$ and $\mathrm{b}$ at $\sqrt{s}=63 \mathrm{GeV}$. The rapidity of the photon is $y=0$, the rapidities of the opposite hadrons $h^{+}$are integrated over the range $\left|y_{h}\right| \leqq 1.0$. The solid (dotted) curves show the result of the calculation for the direct photon production with (without) intrinsic transverse momenta. The dashed curves are the indirect photon contributions $\left\langle q_{T}^{2}\right\rangle \equiv\left\langle q_{T}^{2}\right\rangle_{q, q / h}=0.95(\mathrm{GeV} / c)^{2}$, $\left\langle q_{T}^{2}\right\rangle_{h / q, g}=0.25(\mathrm{GeV} / c)^{2}$.

Concerning the indirect photons we have approximated their contributions by taking the elastic quark-quark and quark-gluon subprocesses and allowing the quark to fragment into a photon with the following fragmentation function as derived in the leading logarithm approximation by Frazer and Gunion [27]

$D_{\mathrm{q}}^{\gamma}\left(z, Q^{2}\right)=e_{\mathrm{q}}^{2} \frac{\alpha}{2 \pi[1-0.72 \ln (1-z)]} \ln Q^{2} / \Lambda^{2}$.

The procedure to obtain the corresponding cross sections is the same as in the case of hadron pairs [16].

Figure 5 shows the cross section $E_{\gamma} d \sigma / d^{3} p_{\gamma} d x_{E}$ for the process $p+p \rightarrow \gamma\left(p_{T}\right)+h^{+}\left(x_{E}\right)+X$ at $\sqrt{s}=$
45 and $63 \mathrm{GeV}^{1}$ ) The photon is emitted at $90^{\circ}$; the rapidity of the hadron is integrated over the range $\left|y_{h}\right| \leqq 1$. In the range $0.4 \leqq x_{E} \leqq 0.8$ the simple expression (4.1) and the one including intrinsic transverse momenta are not very different. In the same range the indirect photon terms contribute at most $25 \%$. For $x_{E}>0.9$ the modifications to $(4.1)$ are large. In order to discuss the effective energy behaviour, we plot in Fig. 6 the cross section times $p_{T}^{4}$ for $p+p \rightarrow \gamma+h^{+}+X$ versus $x_{T}$ at three different energies. The decrease at fixed $x_{T}$ with increasing energy is proportional to $p_{T}^{-1.5}$. This result is quite similar to the $p_{T}$-dependence of CIM contributions, which is predicted to be $p_{T}^{-2.0}[6]$. Therefore a separation of possible CIM contributions based on the $p_{T}$-dependence is difficult, in particular because the normalization of the CIM terms is uncertain.

1 Here and below $h$ denotes the sum of pions and kaons 


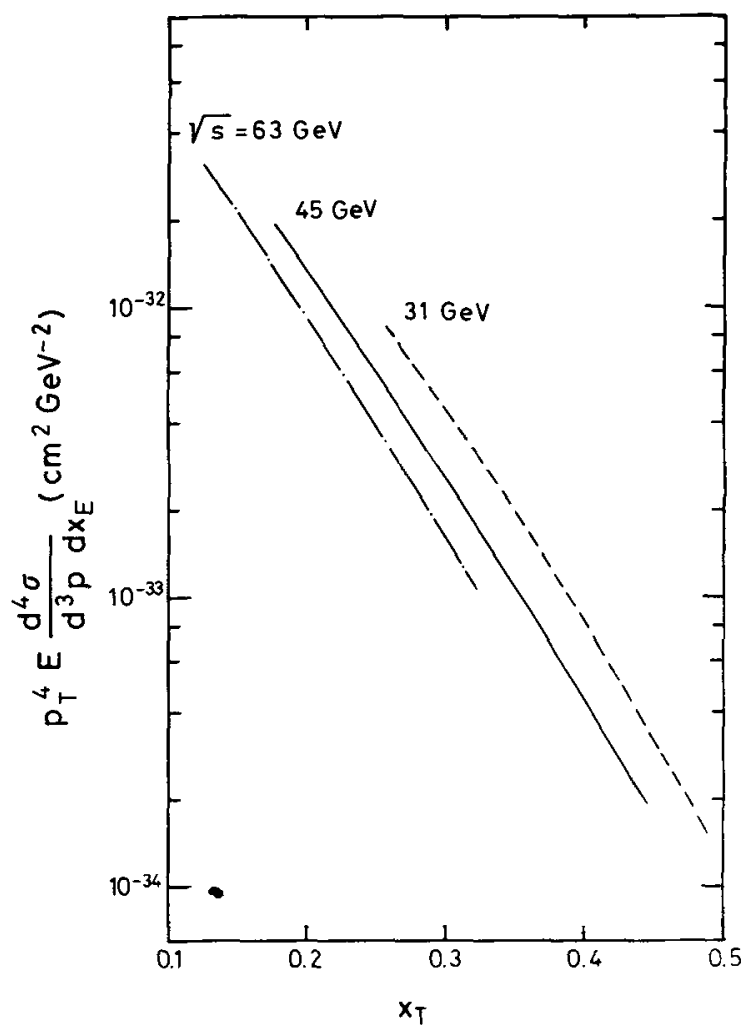

Fig. 6. The $x_{r}$-dependence of the cross section $E d^{4} \sigma / d^{3} p d x_{E}$ times $p_{T}^{4}$ for $p+p \rightarrow \gamma+h^{+}+X$ at $x_{E}=0.6$ for $\sqrt{s}=31,45$ and $63 \mathrm{GeV}$. The rapidities are as in Fig. 5

The situation is similar to large $p_{T}$ hadron production [28].

The predictions for the ratio of positive to negative hadrons on the opposite side $\left(h^{+} / h^{-}\right)$are plotted in Fig. 7 as a function of $x_{7}$ for $\sqrt{s}=63 \mathrm{GeV}$. A measurement of this ratio is important to establish the nature of the recoiling jet. According to (4.1) and (4.3) this jet should in eight out of nine events consist of the fragments of a $u$-quark.

The ratio has been calculated with and without the inclusion of intrinsic transverse momenta. Again as in Fig. 5 the curves with intrinsic transverse momenta continue beyond $x_{E}=1.0$. If (4.1) is applicable then the $h^{+} / h^{-}$ratio should be the same as the one observed in $e(\mu)+p \rightarrow e(\mu)+h^{ \pm}+X$. In Fig. 7 also data from the electron induced reaction [29] and from neutrino-proton reactions [30], which should be compared to the predictions from our fragmentation functions (dashed curves), are plotted. They show that our parametrizations are consistent with the leptoproduction data.

The influence of the indirect photon production on the $h^{+} / h^{-}$ratio is strongest at large $x_{E}$, because $\left(h^{+} / h^{-}\right)_{\text {indirect }} \cong 1.2$ and almost independent of $x_{E}$. We expect that the $h^{+} / h^{-}$ratio is always less than three for $p_{T} \lesssim 8 \mathrm{GeV} / c$.

At this point it is appropriate to make some remarks

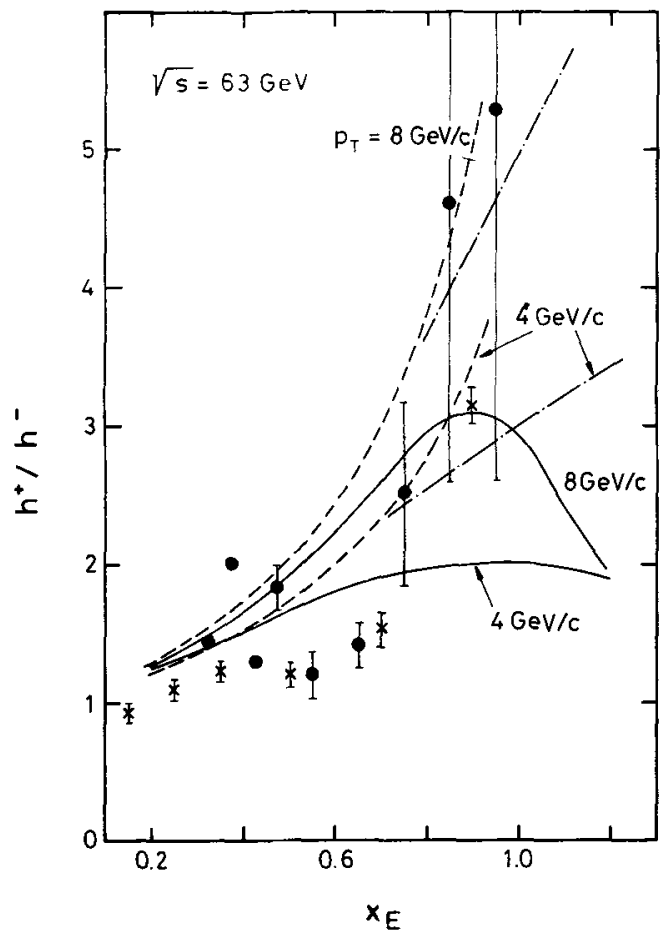

Fig. 7. The ratio of positive to negative hadrons on the opposite side for $p+p \rightarrow \gamma+h^{ \pm}+X$ as a function of $x_{E}$ for $p_{T}=4$ and 8 $\mathrm{GeV} / c$. The dashed-dotted (dashed) curves show the result of the calculation for the direct photon production with (without) intrinsic transverse momenta. The solid curves include the photon bremsstrahlung. The data points are from leptoproduction, [29](-) and $[30](x)$

about the differences to be expected in the QCD model when one triggers on a $\pi^{0}$ instead of a photon. First, the $h^{+} / h^{-}$ratio of hadrons opposite to a $\pi^{0}$ is only between 1.0 and 1.2 in the ISR range and for $4 \lesssim p_{T} \lesssim 10 \mathrm{GeV} / c$. Also the number of hadrons opposite to a $\pi^{0}$ is bigger than opposite to a photon with the same $p_{r}$. There are two reasons for that; the triggered $\pi^{0}$ has not the full momentum to be balanced by the opposite jet, and this jet can be produced from quarks or gluons, where the latter are assumed to have a softer fragmentation function than the quarks. However, counting only the particles with $x_{E} \gtrsim 0.4$ the effects tend to compensate and we find $\left(\gamma+\right.$ charged hadron $/ \pi^{0}+$ charged hadron) $\cong\left(\gamma / \pi^{0}\right)_{\text {single }}$ independent of $p_{T}$. For the same side correlations the following results have been obtained. When the opposite side hadron has $0.4 \leqq x_{E} \leqq 0.8$, the photon is still directly produced in at least $75 \%$ of the events (compared to $90 \%$ in the case of the single particle spectra) and it has therefore the full momentum. In less than $25 \%$ of the events it comes from the bremsstrahlung processes. Under the same conditions the $\pi^{0}$ has, in the ISR range, only about $60-80 \%$ of the same side jet momentum.

From the observation in Fig. 5 that for $0.4 \leqq x_{E} \leqq$ 0.8 , (i) intrinsic transverse momentum effects are negligible and (ii) the indirect photon contributions 
are smaller by at least a factor four, the two-particle cross section near $\theta=90^{\circ}$ is essentially described by (4.1) and (4.3). Thus one can possibly extract $G\left(x, Q^{2}\right)$ from data for $p+p \rightarrow \gamma+$ opposite $\left(h^{+}+h^{-}\right)+X$ with $0.4 \leqq x_{E} \leqq 0.8$ by varying the photon transverse momentum $p_{T}$. The corresponding formula reads

$$
\begin{aligned}
& x G\left(x, Q^{2}\right) \cong 3.3810^{30} \\
& \cdot \frac{p_{T}^{4}\left[(\mathrm{GeV} / c)^{4}\right]}{\alpha_{s}\left(Q^{2}\right) F_{2}\left(x, Q^{2}\right) D_{u}^{\left(h^{+}+h^{-}\right)}\left(x_{E}, Q^{2}\right)} \\
& \cdot \frac{1}{2 \Delta y} \int_{-\Delta y}^{\Delta y} d y_{h} \frac{E_{\gamma} d^{5} \sigma^{p+p \rightarrow \gamma+\left(h^{+}+h^{-}\right)+x}}{d^{3} p_{\gamma} d x_{E} d y_{h}}\left[\mathrm{~cm}^{2} \mathrm{GeV}^{-2}\right],
\end{aligned}
$$
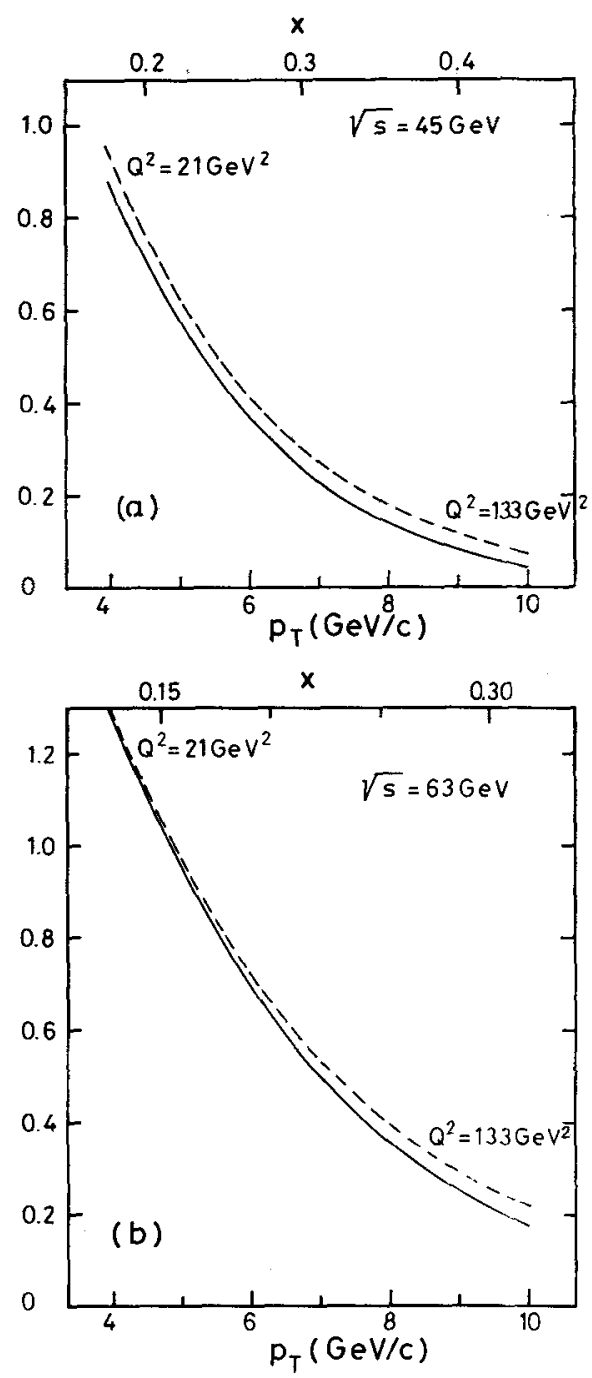

Fig. 8a and $\mathbf{b}$. The dependence of $x G\left(x, Q^{2}\right)$ as a function of $p_{T}$ (and $x=2 p_{T} / \sqrt{s}$ ) at a $\sqrt{s}=45 \mathrm{GeV}, \mathrm{b} \sqrt{s}=63 \mathrm{GeV}$. The dashed curve is calculated from the parametrization (2.1) and (2.2) with $Q^{2}=4 / 3 p_{T}^{2}$, the solid curve is the evaluation of the right hand side of $(4.6)$ for $\Delta y=1.0$ where $x \cong \frac{2 p_{T}}{\sqrt{s}}$, our $Q^{2}=4 / 3 p_{T}^{2}$ at $90^{\circ}$ and $\Delta y \cong$ $0.5-1.0 . F_{2}\left(x, Q^{2}\right)$ is the structure function for deep inelastic lepton-proton scattering.

A comparison of this kind is made in Fig. 8 at two energies $\sqrt{s}=45$ and $63 \mathrm{GeV}$ for $4 \leqq p_{T} \leqq 10$ $\mathrm{GeV} / c$, where we show the input gluon function and the right hand side of (4.6) for $\Delta y=1.0$, including all the background contributions (i)-(iv) in the cross section. Due to the strong scale breaking in the gluon structure function ((2.1) and (2.2)) the right hand side of (4.6) is steeper in $x$ than the gluon structure function at fixed $Q^{2}$.

\section{Summary}

In this paper we have calculated in the framework of QCD the inclusive two-particle cross section for the production of large $p_{T}$ photons produced together with opposite side charged hadrons in $p-p$ collisions. The parametrizations for the structure and fragmentation functions have been taken from our previous work on symmetric hadron pairs [16]. As a check, the single photon and $\pi^{\circ}$ spectra have been calculated and compared to data $[11,12]$ in the range $4 \leqq p_{T} \leqq 10 \mathrm{GeV} / c$ at ISR energies. Agreement is found and confirms our parametrizations, especially the need for a hard gluon structure function.

The two-particle cross section has been computed at $\sqrt{s}=45$ and $63 \mathrm{GeV}$ with and without the inclusion of intrinsic transverse momenta. A comparison between this cross section belonging to a photon and to a $\pi^{0}$ trigger is also made. As in the case of symmetric hadron pairs we have found a simple analytic expression (4.4) for the cross section for direct photon production, which approximates the full model formula with intrinsic transverse momenta in a satisfactory way for $x_{E} \gtrsim 0.4$ and $p_{T} \gtrsim 4 \mathrm{GeV} / c$. This has been checked by Monte Carlo calculations ${ }^{2}$.

The contributions due to the photon bremsstrahlung have been taken into account. The following results have been obtained for $p_{T} \geqq 4 \mathrm{GeV} / c$ :

(i) the Compton subprocess $g+q \rightarrow \gamma+q$ dominates over the fusion process $q+\bar{q} \rightarrow y+g$;

(ii) for $0.4 \leqq x_{E} \leqq 0.8$ the inclusion of intrinsic transverse momenta leads to minor modifications. However, since the cross section for direct photon production vanishes at $x_{E}=1.0$, when the intrinsic transverse momenta are neglected, they become relevant in the region $x_{E}>0.9$;

(iii) for $0.4 \leqq x_{E} \leqq 0.8$ the bremsstrahlung contributions are about $25 \%$ of the direct terms, for $x_{E}>0.9$ they are of the same size;

(iv) the ratio of positive to negative hadrons on

2 The formula (5) in [16] is in fact a good approximation also in this range for hadron pairs and indirectly produced photons 
the opposite side is predicted to increase with increasing $x_{E}$, but because of the indirect photons it stays below three for $p_{T} \leqq 8 \mathrm{GeV} / c$;

(v) because of the strong scale breaking in the gluon structure function a discrimination against CIM terms based only on the $s$ and $p_{T}$ dependence of the two-particle cross section is difficult;

(vi) according to the QCD model there are specific differences expected in the event structure, when one triggers on $\mathrm{a} \pi^{0}$ or a photon.

Following the prescription given in (4.6) one can check the consistency of the QCD-parton model, namely the function obtained should be close to the gluon structure function as extracted from deep inelastic data.

Acknowledgements. Discussions on the experimental results with C.W. Fabjan, K.H. Hansen and B. Lörstad are gratefully acknowledged. We thank E.H. de Groot for a useful discussion.

\section{References}

1. G. Farrar, S.C. Frautschi: Phys. Rev. Lett. 36, 1017 (1976); G. Farrar; Phys. Lett. 67B, 357 (1977)

2. C.O. Escobar: Nucl. Phys. B98, 173 (1975); Phys. Rev. D15, 355 (1977)

3. H. Fritzsch, P. Minkowski: Phys. Lett. 69B, 316 (1977)

4. F. Halzen, D. Scott: Phys. Rev. Lett. 40, 1117 (1978); Phys. Rev. D18, 3378 (1978); Phys. Rev. D21, 1320 (1980)

5. C.H. Llewellyn Smith: Phys. Lett. 79B, 83 (1978)

6. R. Rückl, S.J. Brodsky, J.F. Gunion: Phys. Rev. D18, 2469 (1978)
7. R.D. Field; Proceedings of the XIX Int. Conf. on High Energy Physics, p. 743. Tokyo; 1978

8. A.P. Contogouris, S. Papadopoulos, M. Hongoh; Phys. Rev. D19, 2607 (1979)

9. S. Petrarca, F. Rapuano: Phys. Lett. 88B, 167 (1979)

10. E. Amaldi et al. : Nucl. Phys. B150, 326 (1979)

11. M. Diakonou et al. : Phys. Lett. 91B, 296 (1980)

12. C. Newman: Talk presented at the XV Rencontre de Moriond, 1980

13. C.W. Fabjan: Proceedings of the EPS Int. Conf. on High Energy Physics, p. 742 Geneva; CERN, 1980

14. M. Diakonou et al. : Phys. Lett. 91B, 301 (1980)

15. B.S. Nielsen : Talk presented at the XV Rencontre de Moriond, 1980

16. R. Baier, J. Engels, B. Petersson : Z. Phys. C, 2, 265 (1979)

17. M. Jacob, Proceedings of the EPS Int. Conf. on High Energy Physics, p. 473; Geneva; CERN 1980; P. Darriulat: Ann. Rev. Nucl. Sci. (to be published)

18. J.G.H. de Groot et al. : Phys. Lett. 82B, 45 (1979); Z. Phys. C. 1, 143 (1979)

19. A.J. Buras: Rev. Mod. Phys. 52, 199 (1980)

20. C. Kourkoumelis et al. : preprint CERN- EP/80-07 (1980)

21. A.L.S. Angelis et al. : Phys. Lett. 79B, 105 (1978)

22. R.D. Field: Phys. Rev. Lett. 40, 997 (1978)

23. A.P. Contogouris, R. Gaskell, S. Papadopoulos: Phys. Rev. D17, 2314 (1978); J. F. Owens, E. Reya, M. Glück: Phys. Rev. D18, 1501 (1978)

24. P. Aurenche, J. Lindfors: preprint CERN-TH 2768 (1979)

25. R.K. Ellis, H. Georgi, M. Machacek, H.D. Politzer, G.G. Ross, Nucl. Phys. B152, 285 (1979)

26. R.P. Feynman, R.D. Field, G.C. Fox: Phys. Rev. D18, $3320(1978)$

27. W.R. Frazer, J.F. Gunion: Phys. Rev. D20, 147 (1979)

28. D. Jones, J.F. Gunion: Phys. Rev. D19, 867 (1979); J.F. Gunion, B. Petersson; Preprint UCD-79-5 (1979)

29. G. Drews et al. : Phys. Rev. Lett. 41, 1433 (1978)

30. N. Schmitz: Talk presented at the Int. Symposium on Lepton and Photon Interactions at High Energies, Fermilab 1979. MPI-PAE/Exp. El. 80 (1979) 